\title{
A rare encounter with primary cutaneous mucinous carcinoma on cytology
}

\author{
Pooja S. Kala ${ }^{1, *}$, Harish Chandra ${ }^{2}$ \\ ${ }^{\mathbf{1}}$ Assistant Professor, Dept. of Pathology, Sri Guru Ram Rai Institute of Medical and Health Sciences, Dehradun, Uttarakhand, \\ ${ }^{2}$ Additional Professor, Dept. of Pathology and Laboratory Medicine, All India Institute of Medical Sciences, Rishikesh, \\ Uttarakhand, India
}

*Corresponding Author: Pooja S. Kala

Email: poojahimanshul@gmail.com

\begin{abstract}
Primary cutaneous mucinous carcinoma is a rare adnexal neoplasm of eccrine origin. Most of the cutaneous mucinous carcinomas are metastatic, commonly from gastrointestinal tract and breast. Primary cutaneous mucinous carcinoma was first described in 1952. Only about 200 cases are reported in literature till date. Only a couple of case reports describing the cytological features are available. We report a case of a 70 years old male presenting with nodular lesion on cheek, which was cytologically diagnosed as mucinous neoplasm and later on confirmed on histology and histochemistry to be primary cutaneous mucinous carcinoma. A thorough radiological and clinical workup to rule out the possibility of metastatic deposit of mucinous carcinoma was done.
\end{abstract}

Keywords: Primary cutaneous mucinous carcinoma, Eccrine, Metastatic.

\section{Introduction}

Primary cutaneous mucinous carcinoma or primary mucinous eccrine carcinoma is a rarely encountered entity. It was first described in $1952 .{ }^{1}$ Since then, many cases have been reported in literature including a few case series. Breiting LB et al mentioned that only 228 cases were reported by $2008 .^{2}$ The estimated incidence of cases is 0.07 per million person years. ${ }^{3}$ Only a few case reports are available in the literature describing cytological and histological features of this neoplasm. ${ }^{4-7}$ We report a case of an elderly male presenting with nodular lesion on cheek, which was cytologically diagnosed as mucinous neoplasm and later on confirmed on histology to be mucinous carcinoma. Although, histochemistry favoured it being a primary cutaneous mucinous carcinoma, a thorough search for underlying primary was done, absence of which confirmed it to be a primary cutaneous mucinous carcinoma.

\section{Case Report}

Clinical Details: We report a case of a 70 years old male who presented in general surgery out patient department with a nodule over left cheek from past two years. The nodule was gradually increasing in size. The nodule measured $2 \mathrm{~cm}$ in diameter, was well defined and non-tender. The swelling was adhered to overlying skin but free from underlying tissue. No regional lymphadenopathy was present. Systemic examination was within normal limits.

Cytological Features: The patient was sent for fine needle aspiration cytology in the cytology section of the department. The aspirate yielded few drops of mucoid material. The smears prepared were moderately cellular and comprised of cell clusters and singly scattered cells in a background of abundant mucoid material. The cells were mildly pleomorphic and had round to ovoid nuclei, bland chromatin, moderate amount of ill-defined cytoplasm. Nucleoli were discernible in few of the cells. Some of the cells had vacuolated cytoplasm. Few histiocytes and erythrocytes were seen in background. Cytological diagnosis of mucinous neoplasm was rendered. Excision and histopathological examination was advised.

Histopathological Features: The patient was referred to plastic surgery department and excision was done under general anaesthesia. The excised tissue was sent to histopathology section of the department. The tissue was partially skin covered and measured $2 \times 1.8 \times 1 \mathrm{~cm}$. Cut surface showed a circumscribed grey white and homogenous lesion measuring $1.8 \mathrm{x} 1.5 \times 0.8 \mathrm{~cm}$.

Sections examined showed a tumor in dermis and extending into subcutis comprising of islands and nests of tumor cells floating in mucin pools. Fine fibrous stands were seen traversing through the tumor. The tumor cells were cuboidal and showed only mild cytological atypia. Focal duct formation and cribriform pattern was seen at places. The overlying epidermis was thinned out. Adjacent dermal appendages were unremarkable. All margins were free of tumor. The mucin was PAS positive diastase resistant and stained with alcian blue at $\mathrm{pH} 2.5$. Alcian blue stain at $\mathrm{pH} 1$ was found to be negative. It was opined that the features were of a mucinous carcinoma, possibly primary cutaneous mucinous carcinoma. Another likely diagnosis was metastatic deposit of mucinous carcinoma from an unknown primary. PET/CT scan was done and a thorough search for primary was made by clinicoradiological examination, which were negative. Hence, the final diagnosis of primary cutaneous mucinous carcinoma was rendered. 


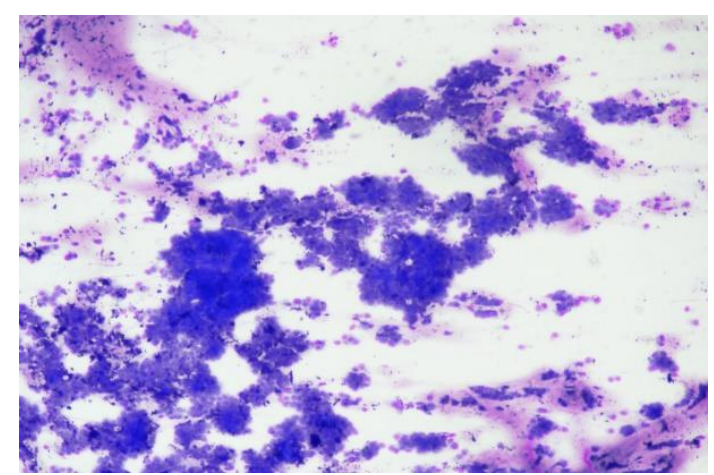

Fig. 1: Primary cutaneous mucinous carcinoma. Cellular smears with cells in clusters and singles lying in a mucoid matrix (MGG, x10).

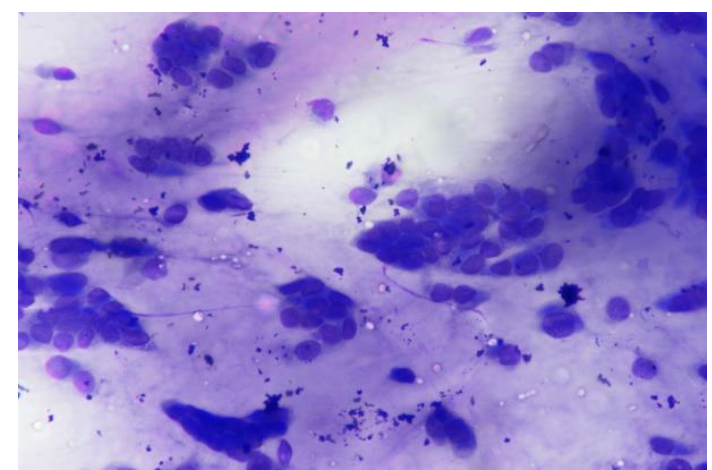

Fig. 2: Primary cutaneous mucinous carcinoma. Mildly pleomorphic cells having round to ovoid nuclei, bland chromatin, moderate amount of illdefined cytoplasm (MGG, $x$ 40)

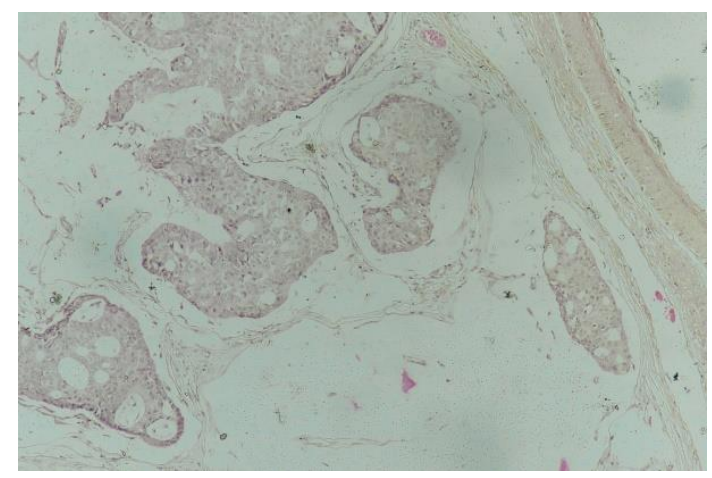

Fig. 3: In the dermis, tumor cells arranged in nests and cribiform pattern are lying in pools of mucin. Thin septa are seen traversing in between. (H\&E, x10)

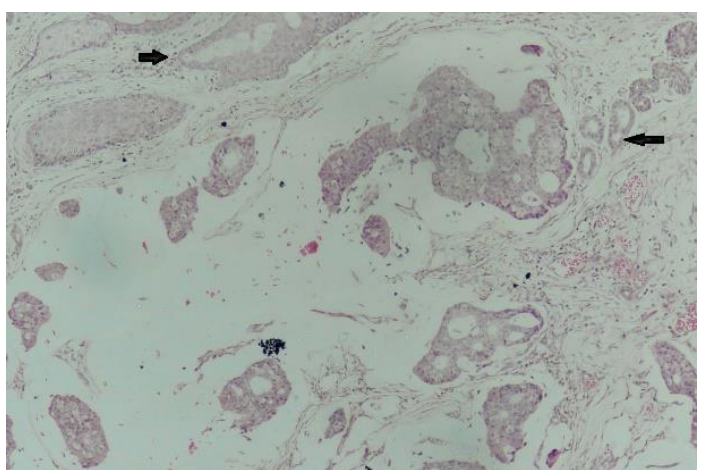

Fig. 4: The adjacent dermal appendages (black arrows) are uninvolved and the tumor is in their close proximity. $(\mathrm{H} \& \mathrm{E}, \mathrm{x10})$

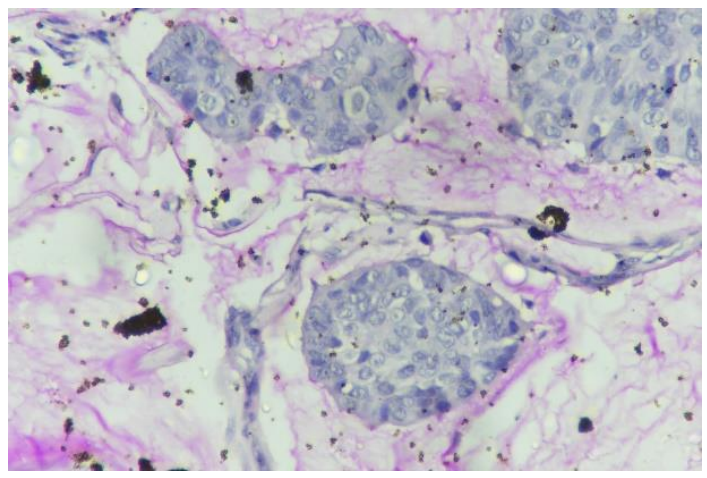

Fig. 5: The mucin stained with PAS stain. (PAS, $\mathbf{x 4 0 )}$

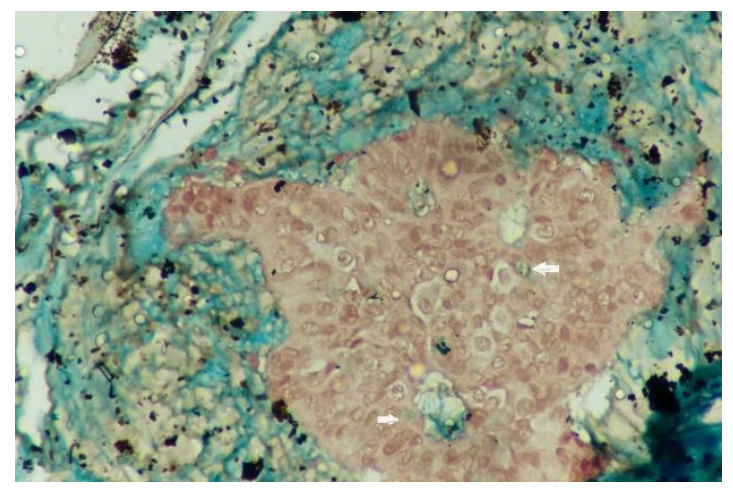

Fig. 6: Mucin stained with the alcian blue at pH 2.5. Intracytoplasmic mucin also present (white arrow). (Alcian blue at pH 2.5, x40)

\section{Discussion}

Primary cutaneous mucinous carcinoma or primary cutaneous colloid carcinoma is a rare adnexal tumor. This tumor generally occurs between fifth to seventh decades of life. Males are slightly more commonly affected than females. ${ }^{8,9}$ Primary cutaneous colloid carcinoma is known to occur on head and neck- eyelids (50\% cases) being the commonest site, followed by face (excluding periorbital area 19.5\%) and scalp $(17 \%) .{ }^{10,11}$ Other uncommon sites are axilla, trunk, lower extremities and genital areas. ${ }^{9}$ These tumours present as solitary, well defined, soft to firm, non 
tender, subcutaneous nodules which gradually increase in size. The size usually varies from 1 to $8 \mathrm{~cm}$, but can be larger as well. ${ }^{12}$

Only meagre work in form of case reports has been published regarding the cytodiagnosis of primary cutaneous mucinous carcinoma. ${ }^{4-7}$ The smears can be of variable cellularity. The cells are present in clusters, cords or in singles and are relatively monomorphic, polygonal to plasmacytoid in appearance with round to oval nuclei, fine chromatin, mild to absent nuclear atypia and moderate to abundant cytoplasm. Background comprise of abundant mucin. ${ }^{4-6}$ The present case also had similar findings. A few mitotic figures can be seen. ${ }^{6}$ Presence of psammoma bodies have also been reported. ${ }^{4}$

Grossly, these are unencapsulated but well circumscribed dermal tumors. The tumor may extend into subcutaneous tissue and in rare instances, may involve overlying epidermis. ${ }^{9}$ The mucinous carcinomas have been described histologically earlier. It comprise of clusters and islands of minimally atypical cuboidal cells floating in large pools of mucin. Delicate fibrous septae are also seen. Cribriform arrangement and focal tubule or gland- like formation are also seen. Intraubular mucin or signs of apocrine secretion can be noted. Periphery of tumor appears more cellular. The cells have abundant cytoplasm which may show vacuolation. Nuclei are round and bland. Rare mitotic figures can be seen. ${ }^{9}$

The mucin in primary cutaneous mucinous carcinoma is sialomucin. This sialomucin is PAS positive, diastase resistant and also shows positive staining with mucicarmine, colloidal iron and alcian blue at $\mathrm{pH} 2.5$ but do not stain with alcian blue at $\mathrm{pH}$ 0.4. This mucin is hyaluronidase resistant while sialidase labile. ${ }^{13}$ As these tumors are of eccrine origin, enzyme histochemistry reveals eccrine enzymes commonly. Electron microscopy shows two types of cells-dark cells and light cells. Dark cells are responsible for secretion of sialomucin. ${ }^{14}$

Differential Diagnosis and Pitfalls: Primary cutaneous mucinous carcinoma is confused with metastatic carcinomas from breast, lung and gastrointestinal tract. The tumors of salivary gland origin, nose and paranasal sinuses, ovary, renal pelvis are also to be excluded. Metastatic mucinous carcinomas are more frequent than the primary ones. It is almost impossible to differentiate the two on histological basis. But several subtle pointers can be helpful. These are- less of mucin, more of epithelial component, larger cell clusters, greater cellular atypia, significant mitotic activity and lack of the fibrous septae in metastatic mucinous carcinoma. ${ }^{8,9}$ Histochemistry can be helpful. Mucin of gastrointestinal tract primary is nonsulfated, neutral and sulphated while that by primary cutaneous mucinous carcinoma is nonsulfated mucin- sialmucin. ${ }^{15}$

Primary cutaneous mucinous carcinoma is CK7, CAM 5.2, CEA, EMA, GCDFP-15, human milk factor globulins, S100, alpha-lactalbumin positive. ${ }^{16}$ CK7 positivity is reported to be $100 \%$, while p63 positivity was $40 \%$. CK5/6 positivity was only $20 \% .{ }^{17}$ ER positivity can be strong while PR positivity can be variable. ${ }^{18}$ CK20 negativity helps differentiates it from metastatic mucinous carcinoma from gastrointestinal tract. ${ }^{19}$ Metastasis from breast primary is even more difficult to exclude. But some authors have stated that a positive immunostain for myoepithelial markers ie p63 and CK5/6 can be helpful to exclude metastatic breast mucinous carcinomas. ${ }^{20}$

In the absence of immunohistochemistry and electron microscopy, one can rely on typical morphology, demonstration of sialomucin and a thorough clinicoradiological work-up to rule out metastasis.

Prognosis: This low grade malignancy has excellent outcomes if wider excision is done. Regional lymph node metastasis is reported to be $4 \%$ to $10 \%$, widespread metastasis only in $3 \%$ cases..$^{21,22}$ Metastasis to lung and extensive bone invasion has also been reported..$^{18}$ Recurrence rate of $30 \%$ has been reported. ${ }^{21}$ Multiple recurrences are known to occur. This may be because of satellite lesions which can be found in such cases. ${ }^{8,9}$ A wide excision, Moh's micrographic surgery followed by regular follow up should be advocated, as adjuvant therapies like chemotherapy and radiotherapy may not help in these tumors. Younger age, Asian background, tumor on the trunk, larger tumors and longer followup periods are factors associated with poorer outcomes. ${ }^{11}$

\section{Conclusion}

Primary cutaneous mucinous carcinomas are rare tumors. Although the cytodiagnosis of this tumor is difficult, but a knowledgeable mind should keep this entity also amongst the differentials, if a mucinous carcinoma is encountered on fine needle aspiration smears.

\section{References}

1. Lennox B, Pears AG, Richards HG. Mucin secreting tumors of the skin, with special reference to the so-called mixed salivary tumor of the skin and its relation to hidradenoma. J Pathol Bacteriol. 1952;74:865-80.

2. Breiting LB, Christensen L, Dahistrom K, Breiting VB, Kalialis LV. Primary mucinous carcinoma of the skin- a literature review. Ugeskr Laeger. 2008;170(43):3399402.

3. Ming SH, Vigneswaran N, Chong MW. Primary mucinous carcinoma of the skin. Eur J Dermatol. 2010;20(6):826-7.

4. Rekhi B, Deodhar KK, Laskar SG, D’Cruz A. Fine needle aspiration cytology in a rare case of recurrent mucinous carcinoma of skin, displaying psammoma bodies on smears. Diagn Cytopathol. 2015;43:937-40.

5. Reid Nicholson M, Iyengar P, Friedlander MA, Lin O. Fine needle aspiration biopsy of primary mucinous carcinoma of the skin: a case report. Acta cytol. 2006;50(3):317-22. 
6. Kotru M, Manucha V, Singh UR. Cytologic and histologic features of primary mucinous adenocarcinoma of skin in the axilla: A case report. Acta cytol. 2007;51:571-74.

7. Rege J, Shet T. Aspiration cytology in diagnosis of primary tumors of skin adnexa. Acta Cytol. 2001;45:71522.

8. Requena L, Kiryu H, Ackerman AB. Neoplasms with apocrine differentiation. Lippincott Williams \& Wilkins: Philadelphia; 1998.

9. LeBoit P.E., Burg G., Weedon D, Sarasain A. (Eds.). World Health Organization Classification of Tumours. Pathology and Genetics of Skin Tumours. Lyon: IARC Press; 2006.

10. Snow SN, Reizner GT. Mucinous eccrine carcinoma of the eyelid. Cancer. 1992;70:2099-104.

11. Kamalpour L, Brindise RT, Nodzenski M, Bach DQ, Veledar E, Alam M. Primary cutaneous mucinous carcinoma: A systematic review and meta-analysis of outcomes after surgery. JAMA Dermatol. 2014;150(4):380-4.

12. Kavanagh GM, Rigby HS, Archer CB. Giant primary mucinous sweat gland carcinoma of the scalp. Clin Exp Dermatol. 1993;18:375-7.

13. Weedon D, Strutton G. Tumors of cutaneous appendages. In: Skin pathology. 2nd ed. St Louis: Elsevier Ltd; 2002.

14. Wright JD, Font RL. "Mucinous gland adenocarcinoma of eyelid: A clinicopathologic study of 21 cases with histochemical and electron microscopic observations." Cancer. 1979;44:1757-68.

15. Martinez SR, Young. "Primary mucinous carcinoma of the skin: A review." Int J Oncol. 2005;2:2.
16. Bellezza G, Sidoni A, Bucciarelli E. "Primary mucinous carcinoma of the skin." Am J Dermatopathol. 2000;22:166-70

17. Levy G, Finkelstein A, McNiff JM.

"Immnohistochemical techniques to compare primary vs. Metastatic mucinous carcinoma of the skin." J Cutan Pathol. 2010;37:411-5.

18. Hanby AM, McKee P, Jeffery M, Grayson W, Dublin E, Poulsom R, et al. "Primary mucinous carcinomas of the skin express TFF1, TFF3, estrogen receptor, and progesterone receptors." Am J Surg Pathol. 1998;22:1125-31.

19. Eckert F, Schmid U, Hardmeier T, Altmannsberger M. “ Cytokeratin expression in mucinous sweat gland carcinomas: an immunohistochemical analysis of four cases." Histopathology. 1992;21:161-5.

20. Qureshi JS, Salama ME, Chitale D, Bansal I, Ma CK, Raju U, et al. "Primary cutaneous mucinous carcinoma: Presence of Myoepithelial cells as a clue to the cutaneous origin." Am J Dermatopathol. 2004;26:353-8.

21. Segal A, Segal N, Gal A, Tumuluri K. "Mucinous sweat gland adenocarcinoma of the eyelid- current knowledge of a rare tumor." Orbit. 2010;29(6);334-40.

22. Wright JD, Font RL. "Mucinous gland adenocarcinoma of eyelid: A clinicopathologic study of 21 cases with histochemical and electron microscopic observations." Cancer. 1979;44:1757-68. 